\title{
Perceptions and Barriers to Contraceptive Use among Adolescents Aged 15 - 19 Years in Kenya: A Case Study of Nairobi
}

\author{
Joyce Kinaro', Murungaru Kimani' ${ }^{2}$, Lawrence Ikamari², Elias H. 0. Ayiemba ${ }^{3}$ \\ ${ }^{1}$ Monitoring and Evaluation, IntraHealth International, Nairobi, Kenya \\ ${ }^{2}$ Population Studies and Research Institute, University of Nairobi, Nairobi, Kenya \\ ${ }^{3}$ Department of Geography and Environment Studies, University of Nairobi, Nairobi, Kenya \\ Email: jwkinaro@gmail.com, jkinaro@yahoo.com
}

Received 15 December 2014; accepted 2 January 2015; published 15 January2015

Copyright (C) 2015 by authors and Scientific Research Publishing Inc.

This work is licensed under the Creative Commons Attribution International License (CC BY).

http://creativecommons.org/licenses/by/4.0/

(c) () Open Access

\begin{abstract}
Even after the launch of family planning program in Kenya, in 1967, contraceptive use among adolescents has remained below 10 percent while child bearing has increased from 2 percent at age 15 to 36 percent at age 19. To understand the roles of perceptions and barriers on contraceptive use, a mixed method study design was applied to a conceptual framework that operationalized these concepts using data from different sources of social interactions in Nairobi, Kenya. Quantitative data were collected from 1119 adolescents aged 15 - 19 years. Qualitative data were collected from adolescents, adolescents' parents and their school teachers. The later were analyzed and interpreted together with the results from cross-tabulations and logistic regression in order to understand the roles of perceptions and barriers. Contraceptive use was found to be 8.6 percent among the adolescents. Overall, unfavourable perceptions among adolescents, parents and teachers seemed to have contributed to low contraceptive use. Unfavourable perceptions played greater role compared to barriers such as sexual partner communication, opinion on adolescents to use a contraceptives and ability to seek contraceptives. Qualitative data show that teachers and parents lack adequate information and skills to discuss sexuality issues. Linking data among adolescents, parents and teachers help to understand the roles of perceptions and barriers to contraceptive use in Kenya. The findings suggest capacity building of teachers and parents on accurate sexuality information for adolescents while services should be youth-friendly.
\end{abstract}

\section{Keywords}

Contraceptive Use, Perceptions and Barriers, Sexuality Information 


\section{Introduction}

Low contraceptive use among adolescents has received global attention with numerous reports in the recent past. Despite the risks associated with early pregnancies, various surveys indicate that sexually active 15 - 19 year old adolescents rarely use contraceptives [1]. In Kenya, only 19.6 percent of currently married adolescents aged 15 19 years use a modern contraceptive while only 5 percent of all adolescents in the country aged 15 - 19 years currently use any modern method of contraceptives [2]. Although knowledge on family planning (FP) is above 98 percent in Kenya and use of contraceptives among currently married adolescents below 20 years old is only 19.6 percent, about 36 percent of youth give birth before reaching 19 years [2].

High adolescent fertility influences population growth and has the potential to contribute to a youthful age structure. According to the 2009 population census, over 55 percent (21.6 million) of the population is below 25 years old [2]. The youthful age structure in turn presents socio-economic and health challenges for the country. Early and unprotected sexual activities expose young people to pregnancy-related health complications and sexually transmitted infections including HIV/AIDS [1]. Inadequate knowledge of the role of condoms and hormonal contraceptives on the prevention of both sexually transmitted diseases and pregnancy continues to expose adolescents to risky sexual behaviour. To respond to the challenges of high adolescent fertility, it is crucial to understand the underlying causes of low contraceptive use among the adolescents.

Although contraceptive use among adolescents is low, in the Kenyan context, little is known and documented about the perceptions of the adolescents regarding access to, and use of contraceptives. To develop more responsive interventions that address the problem of low use of contraception among adolescents, it is crucial to understand adolescents' perceptions and the contributing barriers. In addition, it is important to compare the effects of both barriers and perceptions on contraceptive use among adolescents.

Perceptions about contraceptive use are influenced by information adolescents receive from the family, school and the media [3]. However, a lot of sexually-related information has been found to be inaccurate, ambiguous and sometimes misleading; this has a negative impact on sexual behaviour [4]. In addition, there is no clear guidance on the method or language to use when discussing sexuality issues with adolescents, leaving messages passed, to individual interpretations [4]. Sexually explicit content that is without pregnancy prevention messages has also been found to foster negative attitudes and beliefs about unprotected sex among adolescents [5]. Sex decisions among adolescents are derived from insufficient knowledge. For example, in studies carried out in Bangladesh and Nigeria, adolescent females believed that they could not get pregnant if they washed their genitalia or jumped up and down after intercourse [6] [7]. To respond to the challenges of the effects of perceptions on contraceptive use, it is important to understand the content of the messages/information on sexual matters and contraception that the adolescents receive while at school and at home through their teachers, fellow students, parents and other family members.

The social and physical environment also affects adolescents' perceptions on reproductive health and contraceptive use. Maternal approval, for example, has been associated with a higher probability of contraceptive use among adolescents [8] [9]. However, in many traditional cultures, sexual activity is sanctioned only in marriage. With the breakdown of social structures traditionally used to educate young people on sexuality in Africa, adolescents are becoming sexually active without proper guidance [10]. For example, in a study conducted in Nigeria, only 39 percent of parents surveyed had discussed issues of sex with their children in the year preceding the study [10].

Lack of quality youth-friendly services and information is another problem associated with low use of contraceptives among the youth. In Kenya, only 7 percent of public health facilities provided youth-friendly services [11] [12]. Most of the clinics in Kenya open from Monday to Friday between 8.00 am to 5.00 pm and are therefore inaccessible to adolescents who are likely to be in school during these times [13].

The objective of this study is to contribute to a better understanding of low contraceptive use among adolescents aged 15 - 19 years through identifying perceptions and barriers and their roles on contraceptive behaviour among adolescents in Kenya. The specific objectives of the study were to establish and document perceptions that influence contraceptive use among adolescents aged 15 - 19 years and; identify barriers affecting the use of contraception among adolescents whether in school, out of school, married or unmarried.

Specifically we tested six hypotheses of both perceptions and barriers that: higher knowledge of a contraceptive method is associated with higher levels of contraceptive use; parental approval of contraceptive use is associated with higher contraceptive use; partner communication on contraceptives is associated with contraceptive 
use; marital status is associated with higher contraceptive use; youth friendly and supportive environment is associated with higher contraceptive use and; higher knowledge of risks of unprotected sex is associated with increased contraceptive use.

\section{Data and Methodology}

\subsection{Data Collection and Process of Sampling}

The primary unit for sampling in this study was the household. An inclusion criterion for the sample was 15 - 19 years old males and females in Nairobi. Adolescents who were born before beginning of July 1990 or after June 1995 and those who were not resident in Nairobi were excluded. The sampling frame was the total projected population of adolescent males and females aged 15 - 19 years in Nairobi [14], based on the 2009 Kenya Demographic and Health Survey (KDHS) enumeration clusters. To determine the number of adolescents to interview, a probability sample using a normal sampling distribution was used for precision. Using this approach a sample size of 1169 adolescents' boys and girl was estimated to be adequate. To be able to estimate the number of clusters to be included in the study, the projected number of adolescents (280,400 in Nairobi from the 1999 census) was proportionally allocated among 4476 enumeration clusters containing 651,861 households. Systematic random sampling steps were carried out to proportionally identify the size of 1169 adolescents required for the study. The listing of all households in the 22 clusters randomly selected included cluster identification, names of all occupants and their relationship with the head of the household, age and sex.

The listing also included whether adolescents were attending school and for those attending school in Nairobi, the name of the school. Information on school attendance was useful to identify school teachers to participate in the in-depth interviews (IDIs). The number of adolescents aged 15 - 19 years was selected proportionately according to the cluster population size.

Both quantitative and qualitative data was collected for the study. Triangulation of information enhanced cross checking for internal consistency or reliability. The strategy was used to capture a more complete holistic and contextual portrayal by parents, teachers and adolescents themselves. Triangulation was also used to enrich understanding by allowing new and deeper dimensions to emerge, dissimilar results to emerge and provided an opportunity to reconcile the differences that enriched explanations for divergent results. The qualitative data was collected from the parents and teachers of the adolescents during in-depth interviews. Additional qualitative data was collected from the adolescents during focus group discussions (FGDs). Main qualitative questions used for IDIs among parents and school teachers, and FGDs were to establish the nature and extent of discussions on sexuality and contraceptive use held in school, by family members, and messages passed during these discussions.

Quantitative data were collected from the adolescents-in-school, out-of-school, married and unmarried using a structured questionnaire which was administered during interviews. The interviews were used to collect data that influence contraceptive use among adolescents aged 15 - 19 years and barriers affecting the use of contraception among adolescents. To measure perceptions the respondents were asked the following four questions: How would your guardian/parents feel about your using birth control if you were not married? What would you say is your opinion about unmarried youth using a contraceptive method to avoid getting pregnant? If you wanted to, could you yourself get a contraceptive method? Would you say that you know how contraceptives are used? How often have you discussed contraceptives with your partner in the past one year? Four barrier questions were asked as follows: If you wanted to use a contraceptive method today, would you know where to get them? If you wanted to use contraceptives but you are not using, what are the reasons? If you used a contraceptive before but you are not using it now, what were the reasons?

Data collection took place during school holidays to ensure in-school adolescents participated in the study. The total number of eligible adolescents in these households was 1769 and 1169 were expected sample to be interviewed out of which 1119 representing 96 percent were successfully interviewed. The adolescent females comprised 53.8 percent while the rest were males. Six FGDs were conducted with in-school and out-of-school adolescents. A total of 137 parents and 42 teachers from 40 schools were interviewed in the in-depth interviews. It was a challenge to mobilize adequate numbers of sexually active adolescents living in the same administrative division and therefore the respondents willing to attend FGDs were requested to meet in a common place convenient to them. The information collected was sensitive as it focused on sexuality and therefore, it was not possible to cross-check across causal inferences due to the social desirability bias. To overcome the limitation, 
the study used multiple data collection methods to allow for more probing and validation of information.

The Nairobi University Board of Post Graduate Studies approved the proposal for this study. Permission to conduct the survey was given by the National Council for Science and Technology Ethics and Protocols Board. Kenya National Bureau of Statistics (KNBS) gave permission to use the demographic and health survey enumeration areas. The Nairobi Provincial Commissioner (PC) approved data collection in the community. The respondents were reassured that information collected would remain confidential and no individual names would be used for any purpose and that all information would be pooled for the report.

\subsection{Study Variables}

Knowledge about family planning included awareness and knowledge of how to use contraceptives, source, knowledge of sexuality issues and knowledge of risks of unprotected sex. Respondents were therefore asked whether they had ever heard about contraceptives; how a pregnancy could be prevented; and types of methods of contraception they knew. Computation was carried out using a combination of different types of modern family planning methods listed in the KDHS, namely the pill, intra-uterine device (IUD), injectable, condom, female sterilization and implants. Knowledge of contraceptives was categorized into the following three categories: no knowledge, knew 1 - 2 methods, and knew 3 and more methods. The service environment was assessed to determine quality of services in terms of: clinic open hours, waiting time, ability to obtain services, cost of services, privacy and contraceptive counselling. Eleven items were listed to assess the characteristics of service environment from the perspectives of the respondent.

Perceptions factors were based on: fear of parents and guardians, fear of ridicule from peers, sexual partner communication and ability to get contraceptives by the respondent adolescent. All the respondents were asked whether their parents would approve of them using contraceptives, their opinion on unmarried women using contraceptives for pregnancy prevention and whether they could get a contraceptive if they wanted to. Adolescents who had ever had sex were asked whether they had also ever discussed contraceptives with their partner.

Barriers to contraceptive use arise from factors external to the adolescents. Even when perception to contraceptive is positive, physical barriers could still affect use. Contraceptive barriers were assessed by considering knowledge of contraceptive supply source, clinic open hours, and availability of contraceptive commodities, financial costs/commodity affordability, physical distance, protocols and privacy at the clinic. Respondents were asked whether they knew the source and what would motivate them to collect contraceptives from the source. Respondents were also asked if they would know where to get a contraceptive on the day they wanted to use it. Further, those who knew the source and were current users were asked to give the specific name. This was achieved by asking them to select from a list of clinic characteristics the reasons that would make them collect contraceptives from the selected source. On the other hand those who were not currently using contraceptives were asked what would make them not use contraceptives if they wanted to. To assess the source of contraceptives, respondents who had used contraceptives were asked to give their last source and those who had used contraceptives before and had stopped by the time of the study, were asked why they stopped.

Background factors included socio-cultural environment, demographic and socio-economic factors. Intermediate factors that included knowledge and service provision were used to compare effects of perception and barriers of contraceptive use. Socio-cultural environment variables included family living arrangement (living with parent, living with a relative, living alone and living with a friend).

Values that influence sexuality behaviour are acquired through the socialization process. The first socialization environment is the family, followed by the school and then the wider community. Respondents were asked questions related to their living arrangements. To determine sexuality information passed in different socialization environments, respondents were asked to indicate their most important sources of sexuality information, whether they were currently attending school, and their place of residence.

Socio-economic status influences contraceptive use through ability to access contraceptive commodities, meet travel cost and cost of information materials that create awareness of contraceptives [15]. To assess the level of family wealth, respondents were asked to describe quality of the house, household utilities and materials used in their house construction and whether they lived in the house they were interviewed in, their sources of energy for cooking, ownership of transport means and household items. Items from each section of the question were dichotomized into three and then analysed to generate wealth quintiles categorized as high, middle and low socio-economic status. Demographic factors that were included in this study were: age, sex and marital status 
while service provision environmental variables were: clinic open hours, waiting time, ability to obtain services, cost of services, confidential/privacy of services and contraceptive counselling. Respondents were asked to state their age and sex. On marital status, responses were organized as never married, married, living together, divorced/separated and widowed. A conceptual framework showing the relationship of the above study variables is presented in Figure 1.

The study was guided by a conceptual framework modified from various other studies. The theory of reasoned action applied in the study explains how behaviour is influenced by one's belief that significant other people would not want unplanned pregnancy and by an individual's belief that a preferred action has a beneficial outcome [16]. The cost perspective and Easterlin framework shows that demographic, socio-economic, environmental and cultural factors act through diverse range of intermediate factors that include information and knowledge that influence perceptions and barriers to influence contraceptive use [17]. The sociological framework [18] emphasizes effects of the family in shaping adolescent behaviour and peer influence that affects sexuality activities. The framework further explains that barriers to contraceptive use also arise from mechanisms and institutions that affect individuals accessing reproductive health services [15] [19].

\subsection{Analytical Methods}

Qualitative data analysis involved examining thematic content of memos, using Atlas Ti coding strategy and the continual investigations of the themes for categories, linkages and properties while illustrations of responses used verbatim quotations. Quantitative data analysis included descriptive and multivariate logistic regression models using Statistical Package for the Social Sciences (SPSS). Most of the data analysed were categorical and with associations between dependent, background, intermediate and proximate intervening variables. Multivariate logistic regression was used to estimate odds ratio for each of the independent variables in the model and the combined effects of the explanatory variables in predicting contraceptive use. The results of all variables when analysed together indicated clearly the variables with net significant effects on contraceptive use. The variables with significant effects were used to develop a final model of perceptions and barriers to contraceptive use among the study population.

In line with the study conceptual framework presented above, six models were fitted as follows:

1) Model 1: Background variables

2) Model 2: Perception variables

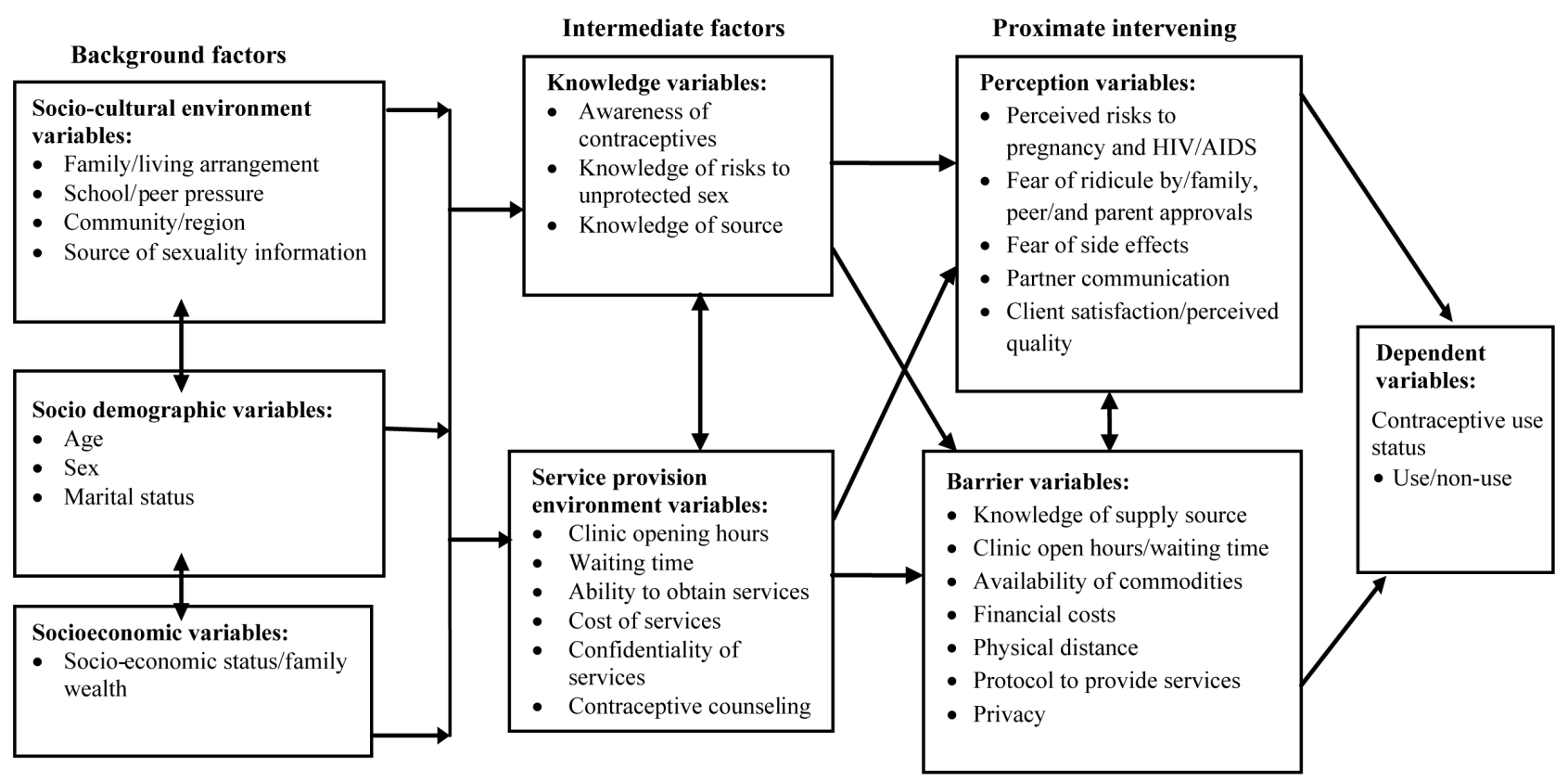

Figure 1. A conceptual framework for studying perceptions and barriers to contraceptive use. Modified from: Fishbein and Ajzen (1980); Twa-Twa (1997); Gage (1998); Magnani et al. (2001); Prince and Hawkins (2007). 
3) Model 3: Barrier variables

4) Model 4: Background and perception variables

5) Model 5: Background and barrier variables

6) Model 6: All the study variables

Qualitative data analysis involved thematic content examination of the memos, using Atlas Ti coding strategy and the continual investigation of the themes for categories, linkages and properties. Verbatim quotations were used to illustrate responses.

\section{Findings}

\subsection{Introduction}

The 1119 adolescents were evenly distributed by age with the least being 19 years old. Both mean and median ages were 17 years. Only 1.7 percent of adolescents were married but this was expected because adolescents aged 15 - 19 years are usually in school. Only 8.6 percent of all adolescents had ever used a contraceptive method as shown in Table 1. Forty six (46) percent of the respondents were males and 54 percent were females. Although 86.6 percent of adolescents were aware of contraceptives, only 43 percent of adolescents knew how to use a contraceptive method. Only 1.7 had high knowledge on the risk to unprotected sex. On perceptions, 65 percents of parents would object to unmarried youth using contraceptives while 23 percent of adolescents approved unmarried youth to use contraceptive methods. In addition, 39.4 percent could get contraceptives for themselves if they wanted them and 7.1 percent communicated about contraceptives with their sexual partners. Overall, barriers factors did not seem to influence respondents to use contraceptives. Knowledge of where to get contraceptives was the main barrier factor found to motivate adolescents to use contraceptives. Qualitative information from both males and females highlights negative perceptions among adolescents regarding service provision and according to in-school adolescents, clinics did not freely provide contraceptives to those below 18 years of age.

\subsection{Differentials in Contraceptive use}

Parental approval was significantly associated with contraceptive use at $\mathrm{p}<0.01$. Contraceptive use increased from 6.2 percent among the adolescents whose parents would object to their using contraceptives to 17.7 percent among adolescents whose parents would not object. Besides the parental approval, the respondents' approval was also found to be important. Contraceptive use rose from 4.1 percent among adolescents who disapprove use by unmarried youth to 23.3 percent among adolescents who approve use by unmarried youth.

The results of the qualitative analysis supported the quantitative results on the association of parental approval and contraceptive use. During IDIs, very few parents indicated they talked about the advantages of contraceptives with their teenage children and cited disadvantages of contraceptives as their main focus of discussion. Most respondents among the parents indicated that if they found their daughters or sons with contraceptives, they would be annoyed. In narratives from FGDs, parents expressed negative perceptions about contraceptive use among adolescents as indicated in the quote below:

Zawadi': "If I found my daughter with pills, I will swing her neck first before I seek to know where she got those (pills) from" (female parent in IDI).

At home, discussions were not structured and parents felt inadequate to discuss sexuality issues as illustrated in some of the excerpts from some parents below:

Jim: "But you know the problem here is that most of the parents do not know about sex education. They do not even know when a child has started her period or menstruation" (male parent in IDI).

Simon: "Using a language that your teenage child will understand...the language that will bring him to the table becomes very hard” (male parent in FGD session).

The unfavourable environment for the use of contraceptives by the respondents extends to the clinics making most adolescents afraid of seeking services because service providers discriminated against them due to age. The following excerpts illustrate the reasons why age is a challenge in contraceptive use:

${ }^{1}$ In this article, names have been exchanged for confidentiality. 
Table 1. Characteristics of the study population according to background variables: Nairobi, Kenya, $2010(\mathrm{~N}=1119)$.

\begin{tabular}{|c|c|}
\hline Characteristics & Percent \\
\hline \multicolumn{2}{|l|}{ Age } \\
\hline 15 & 21.7 \\
\hline 16 & 21.8 \\
\hline 17 & 24.0 \\
\hline 18 & 21.0 \\
\hline 19 & 11.4 \\
\hline \multicolumn{2}{|l|}{ Sex } \\
\hline Male & 46.4 \\
\hline Female & 53.6 \\
\hline \multicolumn{2}{|l|}{ Marital status } \\
\hline Never married & 98.3 \\
\hline Ever married & 1.7 \\
\hline \multicolumn{2}{|l|}{ Socio-cultural environment factors } \\
\hline \multicolumn{2}{|l|}{ Living arrangement } \\
\hline With parents & 75.9 \\
\hline With spouse & 1.3 \\
\hline Elsewhere & 22.1 \\
\hline Not stated & 0.7 \\
\hline \multicolumn{2}{|l|}{ Attending school } \\
\hline In school & 86.6 \\
\hline Out of school & 11.5 \\
\hline No information & 1.9 \\
\hline \multicolumn{2}{|c|}{ Most important source of sexuality information } \\
\hline Teacher & 67.1 \\
\hline Parent & 15.3 \\
\hline Other sources & 17.6 \\
\hline \multicolumn{2}{|l|}{ Place of residence } \\
\hline Central & 5.5 \\
\hline Dagoretti & 4.3 \\
\hline Embakasi & 64 \\
\hline Kasarani & 1.2 \\
\hline Kibera & 6.9 \\
\hline Makadara & 4 \\
\hline Pumwani & 2.7 \\
\hline \multicolumn{2}{|l|}{ Socio-economic status } \\
\hline High & 27.1 \\
\hline Medium & 62.1 \\
\hline Low & 10.8 \\
\hline
\end{tabular}

Source: Primary analysis of the data. 
Joshua: "It is hard to go and buy contraceptives from the shops because when they look at you and see that you are young, they won't give you" (in-school, 16-year-old male respondent).

Josephine: "There are some hospitals where if you go to get those things [contraceptives], they require an identity card from you. At my age, I don’t have such a card” (16-year-old in-school female respondent).

Narratives in this study indicate that the Ministry of Education had introduced life skills curriculum in schools. However, most of the teachers and parents interviewed indicated that sexuality education in school was inadequate and unstructured. Teachers who were expected to carry out counselling activities lacked skills, they were overburdened and they lacked supporting teaching materials.

Veronica: "Nobody is handling the subject adequately. Schools lack proper curriculum, then lack qualified teachers, then lack time. They [schools] lack books and facilities" (female counselling teacher).

On the other hand teachers discouraged sexual activities among students without educating them on risks of unprotected sex. Interview excerpts below illustrate the sexuality education environment in schools:

Charity: “First of all as a teacher, we tend to put what we call don'ts... we do not get in detail. We just say do not do this, but nobody sits down with the students to talk to them about their body changes... their sexual urges... and most of the time we just concentrate... like if it is in this sciences... we just say OK, this is the human body... these are the changes that you go through, that, it [subject] finishes there. If it is religious subject, they (teachers) just say sex is bad... all that... the problem we have is that nobody tells them [students] that in marriage sex is not wrong... nobody tells them that they are students and they will have those urges" (female counselling teacher).

The association between the ability to get contraceptives for oneself and ever use of contraceptive was also significant. Ever use of contraceptive increased from 1.6 percent among adolescents who could not get contraceptives for themselves to 19.0 percent among adolescents who could get contraceptives for themselves. Ever use of contraceptives also increased with sexual partner communication. Based on respondents who ever had sex and therefore had sexual partners, the results indicated that use of contraceptives varied with whether adolescents discussed the issue with sexual partners.

Table 2 shows factors that influence perceptions of contraceptive use among adolescents.

Table 2. Differentials in contraceptive use by Perception variables, Nairobi, Kenya, 2010.

\begin{tabular}{lccc}
\hline \multicolumn{1}{c}{ Factors } & $\begin{array}{c}\text { Ever use } \\
\text { (percent) }\end{array}$ & $\begin{array}{c}\text { Never use } \\
\text { (percent) }\end{array}$ & $\begin{array}{c}\text { Total number } \\
\text { of cases }\end{array}$ \\
$\begin{array}{l}\text { Parent/guardian would approve contraceptive use } \\
\text { for unmarried youth }\end{array}$ & $* * *$ & & \\
$\quad$ Would object & 6.2 & 93.8 & 728 \\
$\quad$ Would not object & 17.7 & 82.3 & 237 \\
$\quad$ Other response & 5.8 & 94.2 & 154 \\
Opinion of unmarried youth to use contraceptives & $* * *$ & & \\
$\quad$ Approve & 23.3 & 76.7 & 266 \\
$\quad$ Disapprove & 4.1 & 95.9 & 838 \\
$\quad$ Not stated & 0.0 & 100 & 15 \\
Can get contraceptives for self if wanted to & $* * *$ & & \\
$\quad$ Yes & 19.0 & 81.0 & 441 \\
$\quad$ No & 1.6 & 98.4 & 615 \\
Not stated & 3.2 & 96.9 & 61 \\
Partner communicates about contraceptives & $* * *$ & & \\
Communicates with sexual partner & 77.2 & 22.8 & 79 \\
Never communicates & 47.7 & 52.3 & 65 \\
Not stated & 0.4 & 99.6 & 975 \\
\hline
\end{tabular}

Notes: $* * *=p<0.01, * *=p<0.05, *=p<0.1$, ns $=$ not significant. 
Among barrier factors, the results indicated that knowledge of the source of contraceptives was significantly associated with contraceptive use at $\mathrm{p}<0.01$. Results indicated that contraceptive use increased by knowledge of source. Use of contraceptives varied from 0.9 percent among adolescents who did not know the source of contraceptives to 13.7 percent among adolescents who knew source. However, during the FGDs with adolescents, they indicated that knowing where to obtain the methods was not enough. Although most respondents seemed to know where contraceptives could be obtained, many of them indicated that they would love to know how the methods work as illustrated below:

Monica: “The provider should explain about pills so that girls can make informed decisions” (out-of school, 18-year-old female adolescent).

Table 3 below shows factors that influence barriers of contraceptive use among adolescents.

Table 3. Differentials in contraceptive use by Barrier variables, Nairobi, Kenya, 2010.

\begin{tabular}{llcc}
\hline Factors & $\begin{array}{c}\text { Ever use } \\
\text { (percent) }\end{array}$ & $\begin{array}{c}\text { Never use } \\
\text { (percent) }\end{array}$ & $\begin{array}{c}\text { Total number } \\
\text { of cases }\end{array}$ \\
\hline
\end{tabular}

\section{Barriers}

If wanted to use contraceptives today would know source

Would know source
Would not know source

Methods available all the time would motivate

\section{No}

Yes

Clinic hours convenient

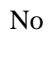

Yes

Free services would motivate

$$
\text { No }
$$$$
\text { Yes }
$$

Distance near enough would motivate

$$
\begin{aligned}
& \text { No } \\
& \text { Yes }
\end{aligned}
$$

Services within reasonable time would motivate

$$
\text { No }
$$$$
\text { Yes }
$$

Friendly protocols/procedures would motivate

$$
\text { No }
$$

\begin{tabular}{|c|c|c|}
\hline 13.7 & 86.3 & 673 \\
\hline 0.9 & 99.1 & 446 \\
\hline \multicolumn{3}{|l|}{$* * *$} \\
\hline 7.9 & 92.1 & 1018 \\
\hline 15.8 & 84.2 & 101 \\
\hline \multicolumn{3}{|l|}{$*$} \\
\hline 8.5 & 91.5 & 1112 \\
\hline 28.6 & 71.4 & 7 \\
\hline \multicolumn{3}{|l|}{ ns } \\
\hline 8.3 & 91.7 & 1069 \\
\hline 14.0 & 86.0 & 50 \\
\hline \multicolumn{3}{|l|}{$* * *$} \\
\hline 4.8 & 95.2 & 836 \\
\hline 19.8 & 80.2 & 283 \\
\hline \multicolumn{3}{|l|}{$* * *$} \\
\hline 8.1 & 91.9 & 1103 \\
\hline 43.8 & 56.2 & 16 \\
\hline \multicolumn{3}{|l|}{$* *$} \\
\hline 8.3 & 91.7 & 1103 \\
\hline 25.0 & 75.0 & 16 \\
\hline \multicolumn{3}{|l|}{ ns } \\
\hline 8.3 & 91.7 & 975 \\
\hline 10.4 & 89.6 & 144 \\
\hline 8.6 & 91.4 & 1119 \\
\hline
\end{tabular}$$
\text { Yes }
$$

Privacy would motivate

No
Yes
Total
Notes: $* * *=\mathrm{p}<0.01, * *=\mathrm{p}<0.05, *=\mathrm{p}<0.1, \mathrm{~ns}=$ not significant.

(1)

\section{3}




\subsection{Multivariate Analysis}

Overall, the most statistically significant variable on the use of contraceptives in the final Model 6 was sexual partner communication. Adolescents who communicated with their sexual partner about contraceptives were 8.3 times more likely to use them compared to those who did not do so; this was significant at $p<0.01$. Other significant variables among perception variables were: ability to get contraceptives for self, opinion of adolescents on contraceptive use and knowledge of how to use a contraceptive method. These variables were all statistically significant at $\mathrm{p}<0.05$. None of the barrier variables were statistically significant. Among the background variables, only two were statistically significant. Ever married adolescents and adolescents out of school were both statistically significant $(\mathrm{p}<0.1)$. When compared with unmarried adolescents, the likelihood of using contraceptives among ever married adolescents reduced from 1.8 more likely to use contraceptives in Model 1 to 0.1 less likely to use in Model 6.

When all the variables were analysed together (Model 6), background factors lost their statistically significant effects on contraceptive use among variables on: age, sex, living arrangement, administrative divisions and source of sexuality information. Barrier factors also lost their significant effect among variables on: knowledge of source of contraceptive methods, physical distance and when services were given within reasonable time. However, none of the perception factors lost their statistical significance on contraceptive use among adolescents. The results demonstrate that perceptions strongly influenced the effects of both background and barrier variables on contraceptive use in the study population. Table 4 shows the results of the multivariate analysis and the odds ratio of both perceptions and barriers to contraceptive use when all variables were tested in the final Model.

\section{Discussion}

The findings of this study were based on a conceptual framework developed to test the influence of perceptions and barriers of contraceptive use among adolescents 15 - 19 years in Nairobi. The test was carried out through six Models of background, perceptions and barrier variables. The study revealed that contraceptive use in Nairobi is low among the adolescents. Although about 87 percent of them were aware of ways to prevent a pregnancy, only 8.6 percent used contraceptives. The other key finding is that perceptions were more significant in the influence of contraceptive use among adolescents compared with barrier factors. In this study, perceptions associated with contraceptive use outcomes were found to be: adolescent approval on contraceptive use, knowledge of how to use contraceptives, ability to get contraceptives for self, and sexual partner communication.

Using both quantitative and qualitative methods, the study revealed that adolescents had the correct perceptions that their parents and guardians objected to contraceptive use a finding similar to other studies [7] [10]. Findings of this study showed that 65 percent of parents would object to unmarried youth using contraceptives. In addition, parents and teachers who participated in IDIs also indicated lack of adequate knowledge and skills to discuss sexuality issues with adolescents. Evidence elsewhere shows that adolescents who perceived their parents to be supportive of condom use were more likely to use them [20]. Results of the regressions analysis however demonstrated that disapproval did not deter use of contraceptives. Such contradictions on the values and practices of sexuality issues among adolescents are similar to those of other studies where adolescents, for example, indicated value for virginity but also said that premarital sex was acceptable [21]. Adolescents may indicate disapproval to conform with culturally acceptable messages but behave differently against the social norms depending on the knowledge they have. According to social learning theory [22], people must believe that avoiding the outcome is beneficial, for example, that using contraceptives will make their lives better in ways that matter to them.

Evidence suggest that partner communication influences contraceptive use as indicated in Pakistan where spousal communication about contraception was associated with contraceptive use [23]. In this study, partner communication was found to significantly influence contraceptive behaviour outcome among adolescents and therefore an important factor to promote for sexual partners.

Knowledge of how to use contraceptives is important. The findings of this study showed that knowledge of how to use contraceptives method significantly influenced use. Current evidence shows that self confidence on contraceptive use influences use [8] and therefore the need for adolescents to have adequate knowledge on how contraceptives work. In this study, ability to get contraceptives was found to be significantly associated with contraceptive use. However, results of FGDs revealed that most adolescents were not able to access contraceptives 
Table 4. Results of the final model indicating odds ratios of the perceptions and barriers on contraceptive use, Nairobi, Kenya, 2010.

Parameters of perception
variables
pregnancy:

$$
\text { No (r) }
$$$$
\text { Yes }
$$

Not stated

Knowledge of how a pregnancy can be prevented:

$$
\text { No (r) }
$$

$$
\text { Yes }
$$

Not stated

Knowledge of contraceptive methods:

Knows 1-2 methods (r)

No Knowledge

Knows 3 and above methods

Knows how to use a contraceptive:

$$
\text { No (r) }
$$

Yes

Not stated

Knowledge of how contraceptives work in prevention of pregnancy and HIV/AIDS:

$$
\text { Low (r) }
$$

High

\section{Moderate}

Knowledge of risks of unprotected sex:

No knowledge (r)

$$
\text { High }
$$

Moderate

$\begin{array}{lc}\text { Odds } & \text { Parameters of perception } \\ \text { ratio } & \text { variables }\end{array}$

Parent approval to use contraceptives:

Other response (r)

56.507

6.105

Would object

Would not object

Opinion of adolescent to use contraceptives:

Disapprove (r)

$0.584 \quad$ Approve

0.0

Can get contraceptives for self if wanted:

No (r)

Yes

0.0

Not stated

2.823 Partner communication on contraceptive use :

Never communicates (r)

Communicates

$26.767^{* *}$

Not stated

0.0

Knows source of contraceptives:

No (r)

\section{Odds}

ratio

Would know source today if wanted to use:

No (r)

$1.790 \quad$ Yes

0.657

2.053 Methods available all the time would motivate:

No (r)

Yes

0.350

$0.208^{* *}$ Convenient clinic hours would motivate:

No (r)

Yes

0.649

$5.021^{* *}$ Free services would motivate:

$7.445 \quad$ No (r)

Yes

1.761

Distance near enough would motivate:

$$
8.316^{* * *} \quad \text { No (r) }
$$

$0.002^{* * *} \quad$ Yes

Services within reasonable time would motivate:

$$
\text { No (r) }
$$

Yes

Friendly protocols/procedures would motivate:

No (r)

Yes

Privacy/confidentiality would motivate:

No (r)

Yes

Yes

1.406

Notes: $\mathrm{r}=$ reference category; na $=$ not available; ${ }^{* * *} \mathrm{p}<0.01, \stackrel{* *}{\mathrm{p}}<0.05, \stackrel{*}{\mathrm{p}}<0.1$; figures without asterisks are not statistically significant. 
when they wanted to use them. The adolescents also indicated that service providers discriminated against them on account of age and in some health facilities, providers demanded national identification cards, thus discouraging adolescents from seeking services. Additionally, shop keepers were reluctant to give contraceptives to adolescents.

In general, the study confirmed that both perceptions and barrier factors are important in influencing contraceptive use among adolescents. The test of significance in the final model of the regression analysis supported two study hypotheses viz.: higher knowledge of how to use a contraceptive method is associated with higher levels of contraceptive use; and, partner communication on contraceptives is associated with contraceptive use. The influence of intervening factors on background factors was consistent with perception formation theory and the effects of barriers on contraceptive use. In support of both perception formation theory [3] [16], and the sociological framework for understanding reproductive health behaviour theory [22], adolescents develop values and knowledge from their immediate physical and social environment, which in turn influence perception and services seeking behaviour. However, barriers encountered during the process of seeking services affect contraceptive use and the experiences adolescents obtained in the external environment during the period of seeking services influence perceptions on use. Consistent with theory [15], the results from this study showed that even if an adolescent had a positive perception on contraceptive use, barriers experienced in the process of obtaining services would influence use.

\section{Conclusion}

Several policy and program issues arise from the findings of this study. The findings confirm the importance of addressing the environmental factors that influenced perception on contraceptive use as a result of sexuality messages passed at the family level and in school. School teachers and parents lacked adequate knowledge and skills to communicate sexual and reproductive health issues. Further, findings demonstrate the importance of developing the capacity of teachers and parents to handle adolescent sexuality issues and pregnancy prevention to reduce negative perceptions on contraceptive use. The study findings also demonstrated the importance of addressing perceptions to influence contraceptive use among adolescents. Service providers were found to discourage contraceptive use on account of age. These findings demonstrated the effects of provider bias on contraceptive use and therefore raise programme issues. There is need to improve service provider skills on quality adolescent sexual and reproductive health services that are youth-friendly. This study also brings out the dilemma of moral tide of adolescent access to contraceptives and the fight against HIV. A further study perhaps on this correlation is needed considering that the children who were born HIV positive in 1990s are currently adults. The trend also pries to the retrogressive or the black patch of assumed age of safety.

\section{Acknowledgements}

This article was developed from a PhD thesis on "Perceptions and Barriers to Contraceptive Use Among Adolescents: A Case Study of Nairobi” conducted through the Population Studies and Research Institute, University of Nairobi. The work was supported by the African Doctoral Dissertation Research Fellowship offered by the African Population and Health Research Centre (APHRC) in partnership with the International Development Research Centre (IDRC) and Ford Foundation.

\section{References}

[1] United Nations Population Fund, Population Issues (2001) Briefing Kit. United Nations Population Fund, New York, 9-17.

[2] Kenya National Bureau of Statistics (KNBS) (2010) Population and Housing Census. Republic of Kenya, Nairobi.

[3] Glanz, K. and Kegler, M. (2002) Concepts of the Social Cognitive Theory Health Behavior and Health Education: Theory, Research and Practice. Wiley and Sons, San Francisco.

[4] Undie, C., Crichton, J. and Zulu, E. (2007) Metaphors We Love by: Conceptualizations of Sex among Young People in Malawi. African Journal of Reproductive Health, 11, 221-235. http://dx.doi.org/10.2307/25549741

[5] Wingood, G., Ralph, J. Harrington, K., Edward, W. and Kim, M. (2001) Exposure to X-Rated Movies and Adolescents' Sexual and Contraceptive-Related Attitudes and Behaviors. Pediatrics, 107, 1116-1119.

http://dx.doi.org/10.1542/peds.107.5.1116 
[6] Nahar, Q., Tuñón, C., Houvras, I., Gazi, R., Reza, M., Huq, N. and Khuda, B. (1999) Reproductive Health Needs of Adolescents in Bangladesh: A Study Report. Centre for Health and Population Research Mohakhali, Dhaka, B Working Paper No. 130. Prime Printers \& Packages, Dhaka.

[7] Otoide, V.O.F., Oronsaye, F. and Okonofua, F.E. (2001) Why Nigerian Adolescents Seek Abortion Rather than Contraception: Evidence from Focus Group Discussions. International Family Planning Perspective, 27, 77-81. http://dx.doi.org/10.2307/2673818

[8] Hulton, L.A., Cullen, R. and Khalokho, S.W. (2000) Perceptions of the Risk of Sexual Activity and Their Consequences among Ugandan Adolescents. Studies in Family Planning, 31, 35-46. http://dx.doi.org/10.1111/j.1728-4465.2000.00035.x

[9] Jaccord, J. and Patricia, J.D. (2000) Adolescent Perceptions of Maternal Approval of Birth Control and Sexual Risk Behavior. American Journal of Public Health, 90, 1426-1430. http://dx.doi.org/10.2105/AJPH.90.9.1426

[10] Izugbara, C.O. (2007) Home-Based Sexuality Education: Nigeria Parents Discussing Sex with Their Children. Journal of Sex Education, 4, 63-79.

[11] Central Bureau of Statistics (Kenya), Ministry of Health (Kenya) and ORC Macro (2004) Kenya Demographic and Health Survey 2003. CBS, Ministry of Health and ORC Macro, Calverton, 210.

[12] National Coordinating Agency and Population Development and Division of Reproductive Health, Ministry of Health (2003) Adolescent Reproductive Health and Development Policy. Tonaz Agenies, Nairobi, 1-37.

[13] Ndhlovu, L., Solo, J., Miller, R. and Miller, K. (1997) An Assessment of Clinic Based Family Planning Services in Kenya: Results from the 1995 Situation Analysis Study. Population Council's Africa Operations Research and Technical Assistance, Nairobi, 19-28.

[14] Kenya National Bureau of Statistics (2007) Kenya Facts and Figures Republic of Kenya. KNBS, Nairobi, 14.

[15] Prince, N. and Hawkins, K.A. (2007) Conceptual Framework for Social Analysis of Reproductive Health. Journal of Health Population Nutrition, 25, 24-36.

[16] Fishbein, M. and Ajzen, A. (1980) Understanding Attitudes and Predicting Social Behaviour. Preventive-Hall, Inc., Englewood Cliffs.

[17] Gage, A., Brandson, J. and Dominique, M. (1993) Sex, Contraception and Childbearing before Marriage in Sub-Saharan Africa. International Family Planning Perspectives, 19, 14-18. http://dx.doi.org/10.2307/2133377

[18] Twa-Twa, J.M. (1997) The Role of the Environment in the Sexual Activity of School Students in Tororo and Pailisa Districts in Uganda. Health Transition Review, 7, 67-81.

[19] Magnani, R.J., Gaffikin, L., Aquino, E.M.L., Seiber, E.E., Almeida, M.C.C. and Lipovsek, V. (2001) Impact of an Integrated Adolescent Reproductive Health in Brazil. Studies in Family Planning, 32, 230-243. http://dx.doi.org/10.1111/j.1728-4465.2001.00230.x

[20] Meekers, D. and Klein, M. (2002) Determinants of Condom Use among Young People in Urban Cameroon. Studies in Family Planning, 33, 335-346. http://dx.doi.org/10.1111/j.1728-4465.2002.00335.x

[21] Ayiemba, E.H.O. (2001) The Effect of Health Education Programmes on Adolescents Sexual Behaviour: A Case Study on Nairobi City Adolescents. African Population Studies, 6, 87-103.

[22] Bandura, A. (1986) Social Foundations of Thought and Action: A Social-Cognitive Theory. Prentice Hall, Englewood Cliffs, 617.

[23] Casterline, J.B., Sathar, Z.A. and Haque, M. (2001) Obstacles to Contraceptive Use in Pakistan: A Study in Punjab. Studies in Family Planning, 32, 95-110. http://dx.doi.org/10.1111/j.1728-4465.2001.00095.x 
Scientific Research Publishing (SCIRP) is one of the largest Open Access journal publishers. It is currently publishing more than 200 open access, online, peer-reviewed journals covering a wide range of academic disciplines. SCIRP serves the worldwide academic communities and contributes to the progress and application of science with its publication.

Other selected journals from SCIRP are listed as below. Submit your manuscript to us via either submit@scirp.org or Online Submission Portal.
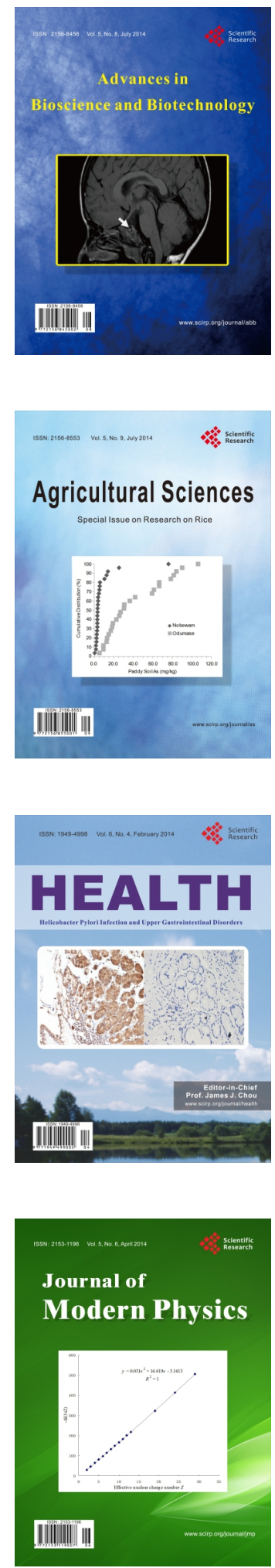
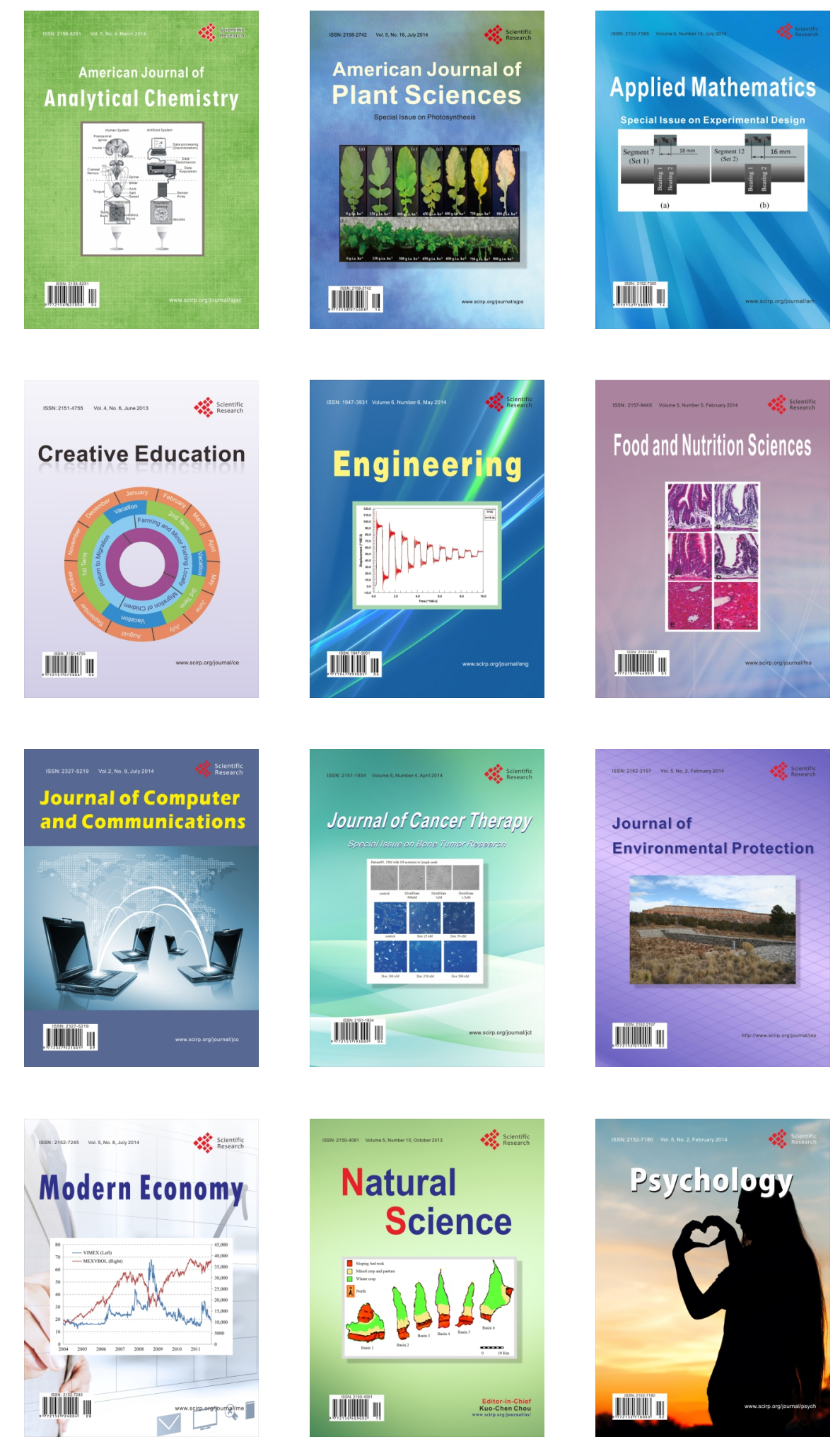